\title{
Novel Nicotinic Receptor Agonist Reduces Hyperalgesia and Allodynia of Neuropathic Pain in Diabetic Rats
}

\author{
Roberto C Debom ${ }^{1,2}$, Margarete M Trachez ${ }^{3,4}$, Gisele Zapata Sudo ${ }^{*}$, Jaqueline S da Silva ${ }^{4}$, Kesley M Oliveira ${ }^{2}$, Ariel L Garcia $^{2}$, Lidiane M Godoy ${ }^{2}$, Ogari C \\ Pacheco ${ }^{2}$ and Roberto T Sudo ${ }^{1,4}$ \\ ${ }^{1}$ Programa de Pós-graduação em Medicina (Cirurgia Geral), Universidade Federal de Rio de Janeiro, Rio de Janeiro, Brazil \\ ${ }^{2}$ Cristália Produtos Químicos e Farmacêuticos Ltda, Itapira, São Paulo, Brazil \\ ${ }^{3}$ Disciplina de Anestesiologia, Universidade Federal Fluminense, Rio de Janeiro, Brazil
}

${ }^{4}$ Programa de Desenvolvimento de Fármacos, Instituto de Ciências Biomédicas, Universidade Federal do Rio de Janeiro, Rio de Janeiro, Brazil

*Corresponding author: Gisele Zapata-Sudo, M.D., Ph.D., Universidade Federal do Rio de Janeiro, Centro de Ciências da Saúde, Instituto de Ciências Biomédicas, Bloco J, Sala 14, Rio de Janeiro, RJ, Brazil, 21941-590 Tel: 55-21-25626478; Email: gsudo@icb.ufrj.br

Rec date: May 06, 2014, Acc date: June 19, 2014, Pub date: June 27, 2014

Copyright: @ 2014 Debom RC, et al. This is an open-access article distributed under the terms of the Creative Commons Attribution License, which permits unrestricted use, distribution, and reproduction in any medium, provided the original author and source are credited.

\begin{abstract}
Diabetic Peripheral Neuropathic Pain (DPNP) has been attributed to abnormal glucose metabolism and oxidative stress. It is among the most common consequence of chronic diabetes and is treated with antiepileptic compounds, antidepressants, and opioids. However, many patients with DPNP experience lack of treatment efficacy and/or adverse secondary effects that impair quality of life. Here we report the identification of a novel agonist targeting neuronal nicotinic Acetylcholine Receptors ( $\mathrm{AAChRs}$ ) that may alleviate neuropathic pain. A new pyrazole analogue, Cris-104, has been observed to produce antinociceptive activity in acute and chronic pain models. In the present study, Cris-104 effects on thermal hyperalgesia and mechanical allodynia were evaluated in a rat DPNP model. Oral administration of Cris-104 (35 mg. $\mathrm{kg}^{-1}$.day ${ }^{-1}$ for 7 days) induced antinociception as evidenced by reductions in thermal hyperalgesia and mechanical allodynia in DPNP rats. The mechanism of antinociception cannot be attributed to a diabetes-alleviating effect because blood glucose levels were not reduced significantly by the treatment. The analgesia could be mediated by activation of nAChRs since Cris-104 is a specific ligand for the nAChR. In conclusion, Cris-104 is a compound with potential to be used as a pain reliever in patients with DPNP.
\end{abstract}

Keywords: Diabetic rats; Nicotinic receptor agonist; Neuropathic pain; Hyperalgesia; Allodynia

\section{Introduction}

Diabetes is a metabolic disease that can induce long-term complications, such as nephropathy, retinopathy, and neuropathy. Diabetes affects approximately 383 million people worldwide currently and its prevalence is expected to increase in the coming decades [1]. Diabetic Peripheral Neuropathic Pain (DPNP) is a highly debilitating sequela of chronic diabetes that affects some $60 \%$ of diabetic patients. It is a consequence of nervous system lesions induced by abnormal glucose metabolism and oxidative stress [2,3]. DPNP is treated with a variety of classes of drugs including anticonvulsants, antidepressants, narcotic analgesics, and local anesthetics. However, many patients are unable to manage their neuropathic pain effectively and the currently prescribed drugs can produce adverse secondary effects that impair quality of life. The pharmaceutical industry is challenged with identifying new pharmacological treatments that can produce greater clinical benefits for patients with DPNP, while reducing side effects and frequency of dosing [4].

The demonstration that the nicotinic cholinergic receptor (nAChR) agonist epibatidine is an effective analgesic agent shifted the attention of pain researchers to the cholinergic system, and to nicotinic subtype receptors in particular [5]. Moreover, there are a variety of neuronal nAChR subtypes in the central and peripheral nervous systems that could thus be targeted with good potential specificity [6]. Neuronal $n A C h R s$ are pentamers (i.e. five subunits) of various combinations of $\alpha(2-9)$ and $\beta(2-4)$ subunits, with the ( $\alpha 4) 2(\beta 2) 3$ configuration being the most prevalent subtype present in the central nervous system. Receptors composed of $\alpha 4$ and $\beta 2$ subunits, collectively referred to as the $\alpha 4 \beta 2$ subtype, are bound by epibatidine, an alkaloid isolated from the skin of a frog (Epipedobates tricolor), which exhibits analgesic activity that is 100 times more effective than that of morphine $[7,8]$. Although epibatidine produces analgesia through activation of $\alpha 4 \beta 2$ subtype nAChRs [7], it also binds muscarinic cholinergic receptors, resulting in side effects such as bradycardia and hypotension [8].

Based on the importance of activation of nicotinic receptors as target for drugs acting to relieve pain, we conducted structure-based design of new ligands for nAChR using the Bionet Screening Compunds Program (Key Organics). One hundred compounds with molecular similarity with epibatidine which could act in the central nervous system were selected to the docking study. Thus, molecular docking was carried out to predict binding mode and orientation of compounds at the active sites of nAChR. Glide XP program successfully docked epibatidine and its derivatives into the active sites of $\alpha 4 \beta 2$ receptor subtype structure $[9,10]$. Docking scores showed that some analogues had high binding affinity to the nicotinic receptor. The most promising derivative which was designated as Cris-160 was found to dock into the active site of nAChR with fitness XP G-score of- $18.08 \mathrm{Kcal} / \mathrm{mol}$. The docking complex was analyzed and it formed one hydrogen bond between Cris-160 and the residue Trp147 of nAChR with bonding distance of $1.77 \AA$ (Figure 1).

The antinociceptive activity was investigated observing the hind paw withdrawal in a hot plate. 
Citation: Debom RC, Trachez MM, Sudo GZ, S da Silva J, Oliveira KM, et al. (2014) Novel Nicotinic Receptor Agonist Reduces Hyperalgesia and Allodynia of Neuropathic Pain in Diabetic Rats. J Diabetes Metab 5: 396. doi:10.4172/2155-6156.1000396

Page 2 of 5

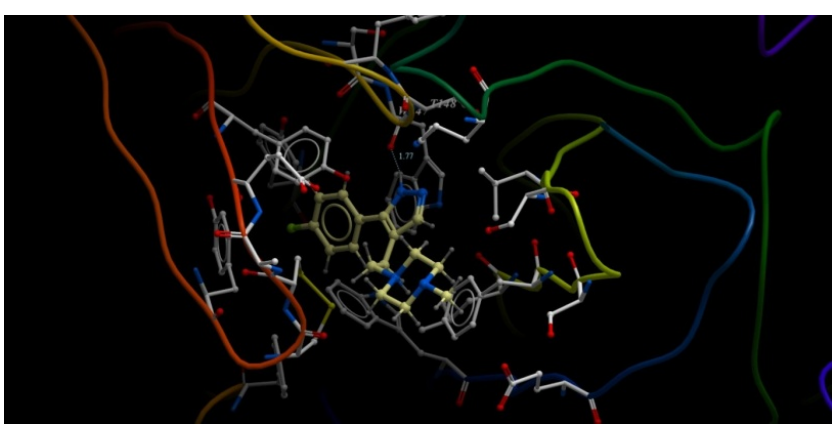

Figure 1: The structural of $\mathrm{nAChR}$ ligand binding domain in complex with Cris-160 obtained by flexible docking

The latency to respond was measured in mice after treatment with either morphine or Cris-160 (12 mg. $\left.\mathrm{kg}^{-1}\right)$. Morphine and Cris-160 produced antinociceptive activity because $5 \mathrm{~min}$ after intraperitoneal administration, the analgesic effect was $68.5 \pm 7.9 \%$ and $47.4 \pm 5.2 \%$, respectively. Thus, Cris-160 induced antinociceptive effect in an acute pain model comparable to morphine and its action could be mediated by the activation of nAChR.

With the aim of developing new selective $\mathrm{nAChR}$ radioligands, structural modification to the prototype molecule Cris-160 were proposed and 14 new analogues were synthesized and subjected to a variety receptor binding assays. The ability of the new analogues to inhibit the binding of agonists to the adenosine, adrenergic ( $\alpha 1, \alpha 2$ and $\beta$ ), canabinoid (CB1 and $\mathrm{CB} 2$ ), dopamine (D1 and D2), gammaaminobutyric acid, gabapentin, glutamate (AMPA and NMDA), histamine $(\mathrm{H} 1-\mathrm{H} 4)$, muscarinic (M1-M5), opiate $(\delta, \kappa, \mu)$, prostanoid (EP2 and EP4), purinergic (P2X and P2Y) and serotonin (5-HT1-5HT5) receptors was tested (performed by MDS Pharma Services, Taiwan). The binding assay experiments implicated only one of the pyrazole analogues as a specific ligand for the nAChR, namely the analogue Cris-104 (Figure 2), which exhibited inhibition of $>60 \%$.

Furthermore, the new pyrazole, Cris-104 (12 mg. $\left.\mathrm{kg}^{-1}\right)$ showed evidence of antinociceptive activity investigated using the hot plate test. The latency to respond with paw withdrawal was increased after intraperitoneal administration of Cris-104 when compared to vehicletreated mice (data not shown).

Based on the evidence that Cris-104 induced antinociceptive effect in acute pain model and had high affinity for the nAChR, this compound was selected for further assessment as a potential analgesic in chronic pain model. The effects of Cris-104 on thermal hyperalgesia and mechanical allodynia, common symptoms of DPNP, were examined in diabetic model rats.

\section{Methods}

The experiments were approved by the Animal Care and Use Committee at Universidade Federal do Rio de Janeiro, Brazil.

\section{Diabetes model}

Male Wistar rats (Rattus norvegicus) weighing 180-220 g received an intravenous (i.v.) injection of streptozotocin (STZ, $60 \mathrm{mg} \cdot \mathrm{kg}^{-1}$ ) dissolved in citrate buffer ( $\mathrm{pH} 4.5$ ) to induce diabetes. Four weeks after induction, a significant increase in blood glucose concentration was observed in STZ-injected animals compared to the non-injected controls. STZ-treated rats with glucose levels $>350 \mathrm{mg} / \mathrm{dL}$ were randomly divided into two groups $(\mathrm{n}=6$ each) and given vehicle (saline) or Cris-104 (35 mg. $\mathrm{kg}^{-1}$ ) orally by gavage once a day for 7 days. Glucose levels were examined with an Accu-Check ${ }^{\bullet}$ Performa glucometer (Roche, Mannheim, Germany) before and weekly after the STZ injection for 4 weeks and at the end of treatment period.

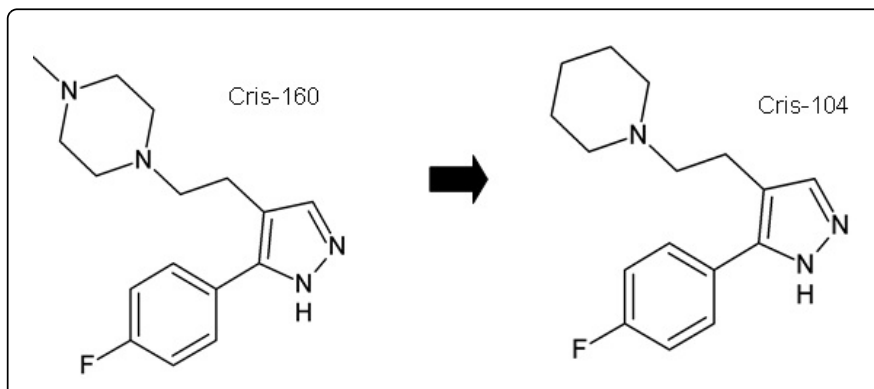

Figure 2: Structural modification proposed for prototype Cris-160. Replacement of 4-methyl-1-piperazinyl present in Cris-160 by 1piperidinyl

\section{Assessment of thermal hyperalgesia and mechanical allodynia}

\section{Pain responsivity}

Thermal hyperalgesia and mechanical allodynia were measured before and 4 weeks after diabetes induction. Animals with a paw withdrawal response significantly lower than that of non-diabetic animals were considered to have neuropathic pain. Paw withdrawal latency and threshold were measured at 1, 3 and 7 days of Cris-104 administration.

\section{Thermal hyperalgesia}

Heat withdrawal latency was assessed by applying a focused radiant heat source (halogen lamp) to the plantar surface of each hind paw through a glass plate $[9,10]$. The light beam was deactivated when the animal lifted its hind paw, allowing measurement of the length of time the paw remained in contact with the heat source. Paw withdrawal latency was determined using a plantar analgesia meter (Model 33; ITC, Woodland Hills, CA, USA). A maximal cutoff of $30 \mathrm{~s}$ was used to prevent tissue damage. Control latency was determined using the average response of three measurements separated by 5 min intervals prior to the STZ injection. Both hind paws were tested. Nociceptive threshold, as determined by heat withdrawal latency was measured prior to and after 4 weeks of diabetic.

\section{Mechanical allodynia}

Withdrawal threshold to pressure applied to the hind paw (in g) was measured with a digital analgesimeter (Model EFF301; Insight, Ribeirão Preto, Brazil) [10,11]. The pressure meter consisted of a hand-held force transducer fitted with a $0.7 \mathrm{~mm}^{2}$ polypropylene tip. Upon paw withdrawal, the pressure was released immediately and the nociceptive threshold was read on a scale. Stimulation of the paw was repeated five times and the readings were accepted if the difference between the highest and lowest measurements was $<10 \mathrm{~g}$. An upper 
limit of $150 \mathrm{~g}$ was used to avoid potential tissue injury in the absence of a response.

\section{Statistical Analysis}

Data are expressed as means \pm standard errors of the mean (SEM). One-way Analysis of Variance (ANOVA) or two-way repeated measures ANOVA was used to analyze the overall effects of treatments. When significant, Dunnett's or Bonferroni's post hoc tests were applied, respectively. Differences were considered statistically significant at $\mathrm{P}<0.05$. All tests were performed using GraphPad Prism (Version 4.0; Graph-Pad Software).

\section{Results}

\section{Blood glucose levels in streptozotocin-induced diabetes in rats}

Blood glucose levels in the Cris-104 group did not differ significantly from levels observed in non-treated diabetic controls. After oral administration of Cris-104, serum glucose level was $557.2 \pm$ $27.3 \mathrm{mg} / \mathrm{dL}$ compared to $511.5 \pm 38.0 \mathrm{mg} / \mathrm{dL}$ measured in the nontreated diabetic rats.

\section{Cris-104 reduced thermal hyperalgesia and mechanical allodynia}

Four weeks after STZ injection, mean paw withdrawal latency (PWL) and paw withdrawal threshold (PWT) of STZ-treated rats were reduced significantly from $12.2 \pm 0.4 \mathrm{~s}$ to $8.9 \pm 0.2 \mathrm{~s}$ and from $38.9 \pm$ $0.6 \mathrm{~g}$ to $27.7 \pm 1.9 \mathrm{~g}$, respectively (Figure $3 \mathrm{~A}$ and $3 \mathrm{~B}$ ), demonstrating the installation of neuropathic pain. As shown in Figure 3A, subsequent daily oral administration of Cris-104 (35 mg.kg-1) increased PWL significantly after $3(12.4 \pm 0.3 \mathrm{~s})$ and 7 days $(12.1 \pm 0.7$ s) of treatment. Additionally, as shown in Figure 3B, Cris-104 increased PWT significantly after 3 days $(27.8 \pm 0.5 \mathrm{~g})$ and 7 days (32.6 $\pm 1.4 \mathrm{~g}$ ) of treatment. Both parameters returned completely too pretreatment level 7 days after suspension of treatment.

\section{Cris-104 in locomotor activity}

Sedative property of Cris-104 was investigated by recording locomotor activity of diabetic rats in an open field. The mobility of Cris-104-treated rats $(113.0 \pm 4.6$ movements $/ \mathrm{min})$ over $60 \mathrm{~min}$ did not differ significantly from that of diabetic rats given only saline $(147.2 \pm 23.5)$ in an open field after 7 days of treatment (Figure 4). Meanwhile, diazepam (10 mg. $\left.\mathrm{kg}^{-1}\right)$, the sedation positive reference treatment, reduced locomotor activity significantly from $147.2 \pm 23.6$ movements/min before injection to $50.7 \pm 6.4$ movements/min after injection.

\section{Discussion}

In the current study, we investigated the antinociceptive activity of a new non-opioid compound in a STZ-induced rat model of diabetes. Symptom of thermal hyperalgesia is commonly observed in diabetic rats submitted to intense lesion of pancreatic $\beta$-cells induced by streptozotocyn (60 mg.kg-1 i.v.) [11,12]. Thermal hyperalgesia and mechanical allodynia are detected after 4 weeks after streptozotocyn administration which suggest the development of diabetic neuropathy. Those animal signs have been useful to test efficiency of drugs for the treatment of neuropathic pain. Daily oral administration of Cris-104 for 7 days induced antinociception in diabetic rats evidenced by reductions in thermal hyperalgesia and mechanical allodynia. Our results suggest that Cris-104 may be a valuable adjuvant compound for treatment of DPNP. The 7 of days Cris-104 treatment did not alter blood glucose levels significantly, suggesting that Cris-104's mechanism is probably not related to an antidiabetic activity per se. additionally, locomotor activity was not affected significantly by Cris-104, indicating that it should not have a sedative effect.
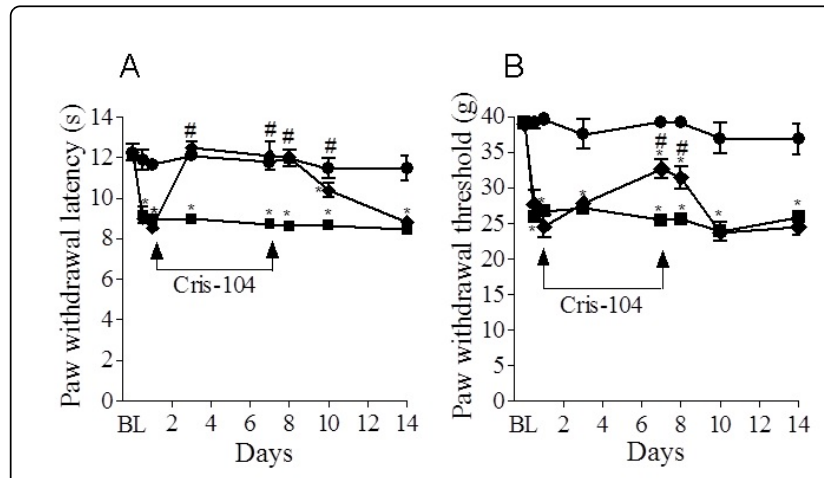

Figure 3: Thermal hyperalgesia (A) and mechanical allodynia (B) of non-diabetic rats $(\bullet, \mathrm{n}=4)$ and diabetic rats treated with either saline $(\bullet, \mathrm{n}=6)$ or Cris-104 $(\bullet, \mathrm{n}=6)$ at dose of $35 \mathrm{mg} . \mathrm{kg}^{-1}$. Treatment was initiated 4 weeks after STZ injection to induce diabetes. Data are mean \pm SEM. ${ }^{*} \mathrm{P}<0.05$ compared to control (BL) \# $\mathrm{P}<0.05$ compared to diabetic + saline

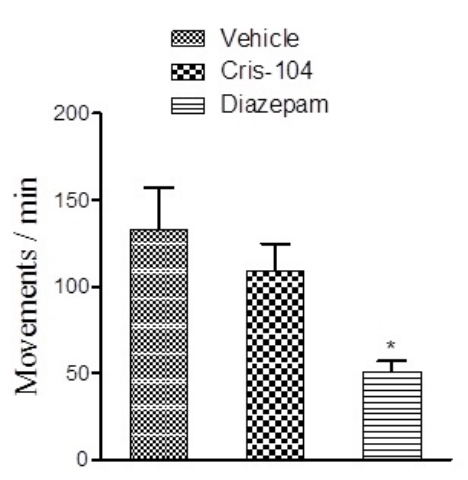

Figure 4: Effect of Cris-104 on locomotor activity in rat. The dose of Cris- 104 was $35 \mathrm{mg}$. $\mathrm{kg}^{-1}$ and diazepam was $10 \mathrm{mg} \cdot \mathrm{kg}^{-1}$. The number of movements was measured for $60 \mathrm{~min}$ after treatment with diazepam and after 7 days of treatment with Cris-104. Values are mean \pm SEM. $n=6{ }^{\star} \mathrm{P}<0.05$ compared to vehicle.

Nicotine-induced antinociception appears to be a complex phenomenon that involves multiple nAChR subtypes depending on the pain type and sites of action. The discovery of epibatidine, a potent $\mathrm{nAChR}$ agonist, was of great importance for treatment of chronic pain. Unfortunately, early tests showed that it had several toxic effects when used clinically [4]. With the aim of introducing new analgesics chemically related to epibatidine, a new compound named ABT-594 was synthetized with the advantages of causing few effects on the 
cardiovascular system [12] and being more potent than morphine [13]. However, intolerable adverse secondary effects (i.e. nausea, vomiting, and dizziness) were observed, terminating development of ABT-594 during phase II clinical trials [14]. With the identification of the structure of epibatidine and using [125I]-epibatidine [15], it was possible to identify the distribution of nAChRs in the brain and the role of a4ß2 subunits, generally considered to be the major neuronal $\mathrm{nAChR}$ in the brain [16].

The $a 4 ß 2$ nAChR subtype exists in two stoichiometric configurations, ( $\alpha 4) 2(ß 2) 3$ and (a4)3(ß2)2, which are found in different brain areas. These two stoichiometries (also called "subtypes" by some authors) have been distinguished by their sensitivity to cytisine in competitive ligand binding assays, with receptors of the (a4)2(B2)3 configuration having greater affinity for cytisine and (a4)3 (ß2)2 having lower affinity for cytosine [17]. Recently, binding assays showed that Cris-104 has a high affinity for $(\alpha 4) 3(ß 2) 2$ receptors $(61 \%$ inhibition) and a low affinity for ( $(4) 2$ (ß2)3 receptors.

The pharmacological differences between receptor subtypes are therapeutically explored through the development and use of drugs that are selective for each receptor. Increasing the selectivity of a drug for a particular tissue or receptor subtype can enable it to produce the desired therapeutic response without additional unwanted effects. Gao et al. demonstrated that the $\mathrm{nAChR}$ agonist varenicline (prescribed for smoking cessation) was effective in a neuropathic pain model in the dose range of 3-10 mg/kg [18] and that at higher doses (two orders of magnitude) may have a non-selective activating effect on multiple receptor subtypes including receptors with $\alpha 4, \beta 2, \alpha 7$, and $\alpha 3$ subunits, and that these other binding activities may contribute to an analgesic effect, but with a low therapeutic index [19].

The mechanisms mediating analgesia via involvement with $\mathrm{nAChRs}$ are poorly understood. Nicotinic receptors are found in the spinal cord dorsal horns, along the ascending and descending nociceptive pathways, and in supraspinal sites. Antinociceptive effects mediated through activation of $\alpha 4 \beta 2 \mathrm{nAChRs}$ have been attributed to enhancement of descending monoaminergic inhibition in the nucleus raphe magnus and locus ceruleus [10] and stimulation of GABAergic and glycinergic inhibitory transmission in the spinal cord [20,21].

The measurement of motor activity in rodents is a fundamental behavioral test in drug safety testing. Several compounds with high analgesic activity, such as morphine, tramadol, and benzodiazepine analgesic-adjuvant compounds can impair motor activity. Daily administration of Cris-104 for 7 days did not have a significant effect on locomotor activity. This result suggests that its analgesic activity is unlikely to impair motor activation through central or peripheral action.

In conclusion, data from the present study suggest that the recently discovered new nAChR agonist Cris-104 is a compound with the potential to be developed into a treatment for the symptoms of DPNP. It appears to work primarily through activation of the (a4)2(B2)3 $\mathrm{nAChR}$, which may enable it to produce pain relief without untenable adverse secondary effects. Currently used medications, such as antiepileptic compounds (gabapentin and pregabalin), antidepressants (amitriptyline and duloxetine), and opioids, have considerable adverse secondary effects that have limited their use [22]. The present results are promising in suggesting that Cris-104 has the potential to be developed into an efficacious treatment for DPNP symptoms without excessive side effects [23-26].

\section{Acknowledgements}

This work has been supported by Cristália Produtos Químicos e Farmacêuticos Ltda, Coordenação de Aperfeiçoamento de Pessoal de Nível Superior (CAPES), Fundação Carlos Chagas Filho de Amparo à Pesquisa do Estado do Rio de Janeiro (FAPERJ), Conselho Nacional de Desenvolvimento Científico e Tecnológico (CNPq).

\section{References}

1. Danaei G, Finucane MM, Lu Y, Singh GM, Cowan MJ, et al. (2011) National, regional, and global trends in fasting plasma glucose and diabetes prevalence since 1980: systematic analysis of health examination surveys and epidemiological studies with 370 country-years and 2.7 million participants. Lancet 378: 31-40.

2. Feldman EL, Stevens MJ, Russell JW, Greene DA (1999) Diabetic neuropathy. In: Current Review of Diabetes. Taylor S (Ed) Current Medicine, Philadelphia: 71-83.

3. Daousi C, MacFarlane IA, Woodward A, Nurmikko TJ, Bundred PE, et al. (2004) Chronic painful peripheral neuropathy in an urban community: a controlled comparison of people with and without diabetes. Diabet Med 21: 976-982.

4. Nightingale S (2012) The neuropathic pain market. Nat Rev Drug Discov 11: 101-102.

5. Badio B, Daly JW (1994) Epibatidine, a potent analgetic and nicotinic agonist. Mol Pharmacol 45: 563-569.

6. Aceto MD, Martin BR, Tripathi HL, May EL, Jacobson AE (1980) Antinociceptive effects of the optically pure stereoisomers of nicotine. Pharmacologist 22:302.

7. Daly JW, Garraffo HM, Spande TF, Decker MW, Sullivan JP, et al. (2000) Alkaloids from frog skin: the discovery of epibatidine and the potential for developing novel non-opioid analgesics. Nat Prod Rep 17: 131-135.

8. Dallanoce C, Matera C, Pucci L, Gotti C, Clementi F, et al. (2012) Synthesis and binding affinity at $\alpha 4 \beta 2$ and $\alpha 7$ nicotinic acetylcholine receptors of new analogs of epibatidine and epiboxidine containing the 7azabicyclo[2.2.1]hept-2-ene ring system. Bioorg Med Chem Lett 22: 829-832.

9. Friesner RA, Banks JL, Murphy RB, Halgren TA, Klicic JJ, et al. (2004) Glide: a new approach for rapid, accurate docking and scoring. 1. Method and assessment of docking accuracy. J Med Chem 47: 1739-1749.

10. Halgren TA, Murphy RB, Friesner RA, Beard HS, Frye LL, et al. (2004) Glide: a new approach for rapid, accurate docking and scoring. 2. Enrichment factors in database screening. J Med Chem 47: 1750-1759.

11. Zapata-Sudo G, Lima LM, Pereira SL, Trachez MM, da Costa FP, et al. (2012) Docking, synthesis and anti-diabetic activity of novel sulfonylhydrazone derivatives designed as PPAR-gamma agonists. Curr Top Med Chem 12: 2037-2048.

12. Levine JH, Buse MG, Leaming AB, Raskin P (1978) Effect of streptozotocin-induced diabetes on hepatic ornithine decarboxylase activity in the rat. Endocrinology 103: 1234-1238.

13. Hargreaves K, Dubner R, Brown F, Flores C, Joris J (1988) A new and sensitive method for measuring thermal nociception in cutaneous hyperalgesia. Pain 32: 77-88.

14. Mendes TC, Antunes F, Trachez MM, Nascimento NM Jr, Fraga CA, et al. (2013) Antihyperalgesic effects of a novel muscarinic agonist (LASSBio-873) in spinal nerve ligation in rats. Clin Exp Pharmacol Physiol 40: 404-411.

15. Vivancos GG, Verri WA Jr, Cunha TM, Schivo IR, Parada CA, et al. (2004) An electronic pressure-meter nociception paw test for rats. Braz J Med Biol Res 37:391-399.

16. Holladay MW, Wasicak JT, Lin NH, He Y, Ryther KB, et al. (1998) Identification and initial structure-activity relationships of (R)-5-(2azetidinylmethoxy)-2-chloropyridine (ABT-594), a potent, orally active, non-opiate analgesic agent acting via neuronal nicotinic acetylcholine receptors. J Med Chem 41: 407-412. 
Citation: Debom RC, Trachez MM, Sudo GZ, S da Silva J, Oliveira KM, et al. (2014) Novel Nicotinic Receptor Agonist Reduces Hyperalgesia and Allodynia of Neuropathic Pain in Diabetic Rats. J Diabetes Metab 5: 396. doi:10.4172/2155-6156.1000396

Page 5 of 5

17. Kesingland AC, Gentry CT, Panesar MS, Bowes MA, Vernier JM, et al. (2000) Analgesic profile of the nicotinic acetylcholine receptor agonists, (+)-epibatidine and ABT-594 in models of persistent inflammatory and neuropathic pain. Pain 86: 113-118.

18. Umana IC, Daniele CA, McGehee DS (2013) Neuronal nicotinic receptors as analgesic targets: it's a winding road. Biochem Pharmacol 86: 1208-1214.

19. Whiteaker P, Jimenez M, McIntosh JM, Collins AC, Marks MJ (2000) Identification of a novel nicotinic binding site in mouse brain using [(125)I]-epibatidine. Br J Pharmacol 131: 729-739.

20. Flores CM, Rogers SW, Pabreza LA, Wolfe BB, Kellar KJ (1992) A subtype of nicotinic cholinergic receptor in rat brain is composed of alpha 4 and beta 2 subunits and is up-regulated by chronic nicotine treatment. Mol Pharmacol 41: 31-37.

21. Pabreza LA, Dhawan S, Kellar KJ (1991) [3H]cytisine binding to nicotinic cholinergic receptors in brain. Mol Pharmacol 39: 9-12.

22. Gao B, Hierl M, Clarkin K, Juan T, Nguyen H, et al. (2010) Pharmacological effects of nonselective and subtype-selective nicotinic acetylcholine receptor agonists in animal models of persistent pain. Pain 149: 33-49.

23. Rollema H, Shrikhande A, Ward KM, Tingley III, Coe JW et al. (2010) Pre-clinical properties of the a4ß2 nicotinic acetylcholine receptor partial agonists varenicline, cytisine and dianicline translate to clinical efficacy for nicotine dependence. Brit J Pharmacol 160:334-345.

24. Bitner RS, Nikkel AL, Bannon AW, Arnwric SP, Decker MW (1997) Supraspinal c-fos induction produced by a novel cholinergic channel modulator analgesic, ABT-594. Soc Neurosci Abstr 23:1200.

25. Rashid MH, Furue H, Yoshimura M, Ueda H (2006) Tonic inhibitory role of alpha4beta2 subtype of nicotinic acetylcholine receptors on nociceptive transmission in the spinal cord in mice. Pain 125: 125-135.

26. Rowbotham MC, Arslanian A, Nothaft W, Duan WR, Best AE, et al. (2012) Efficacy and safety of the $\alpha 4 \beta 2$ neuronal nicotinic receptor agonist ABT-894 in patients with diabetic peripheral neuropathic pain. Pain 153: $862-868$. 